South America (2.3); and countries of Upper-Middle Income (2.8). Regions with higher incidence of young-onset gastric cancer had higher prevalence of alcohol drinking (beta coefficients $(\beta)=0.066,95 \%$ confidence intervals (CI) 0.023 to $0.109, p=0.003)$ and unhealthy dietary habits $(\beta=0.031,95 \%$ CI 0.012 to $0.050, \mathrm{p}=0.001)$, but not smoking $(\beta=0.018$, $95 \%$ CI -0.010 to $0.046, p=0.210$ ) among the young population.

Conclusions The burden of young-onset gastric cancer was substantial in 2020, with higher incidence found in male subjects, Eastern Asia, Central and South America. The associated lifestyle risk factors for young-onset gastric cancer included alcohol drinking and unhealthy dietary habits. To prevent young-onset gastric cancer, lifestyle modifications and early detection by screening for high-risk young individuals could be imperative interventions.

A OS of Patients with Left Colon Cancer

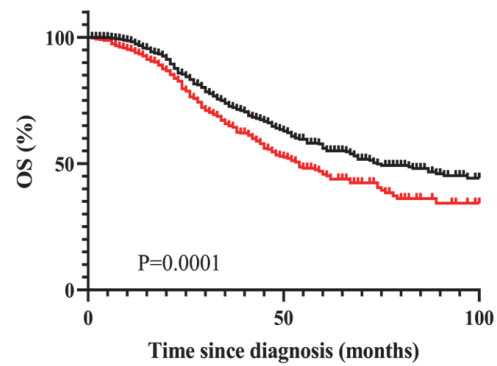

- Male (n=1102)

- Female (n=580)

A

OS of Patients wih Simultaneous Liver Metastases (All Patients, $n=1573$ )

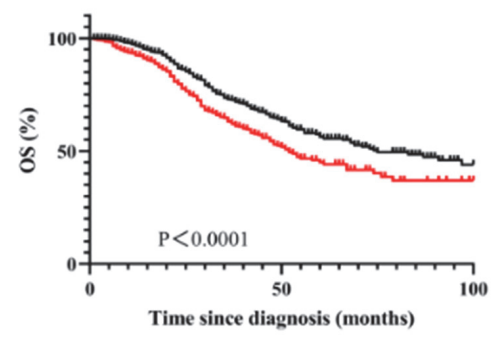

- Male (n=1042)

- Female $(\mathrm{n}=531)$

(a)

Abstract IDDF2021-ABS-0191 Figure 1

Abstract IDDF2021-ABS-0191 Figure 2

A

OS of CRCLM Patients with KRAS ${ }^{+}$ Status (All Patient, $n=461$ )
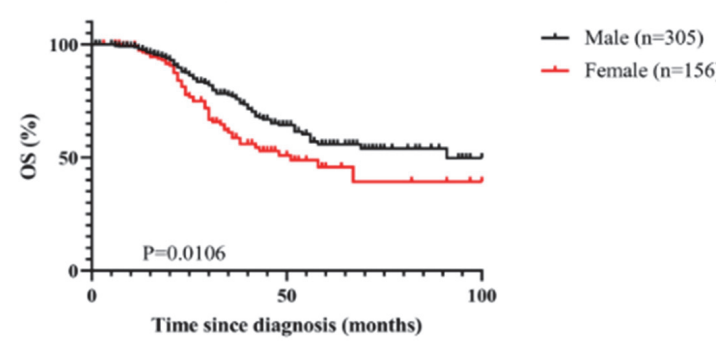

- Female (n=156)
IDDF2021-ABS-0191

GENDER MATTERS: SEX DISPARITIES IN COLORECTAL CANCER LIVER METASTASIS SURVIVAL: A POPULATION-BASED STUDY

Yibin Wu*, Weiqi Xu, Lu Wang. Fudan University Shanghai Cancer Center, China

\subsection{6/gutjnl-2021-IDDF.172}

Background Colorectal cancer with liver metastasis showed a poorer prognosis in colon cancer. The aim of this study was to investigate the impact of sex disparities in survival. The patients were stratified according to sex, age, primary tumor site, KRAS mutant status and metastatic characteristics.

Methods Patients diagnosed with colorectal cancer liver metastasis (CRCLM) between January 2007 and June 2018 at our hospital were identified. Clinical information, tumor character-

B OS of Patients with Right Colon Cancer Metastasis (All Patients, $n=550$ )

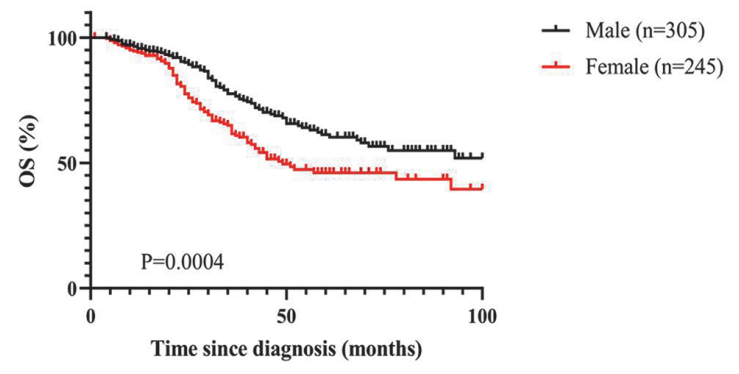

Time since diagnosis (months)

B

OS of Patients wih Metachronous Liver Metastasis (All Patients, $n=1030$ )

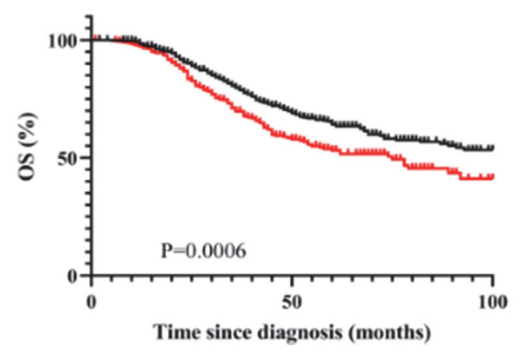

- Male (n=611)

- Female (n-419)

B

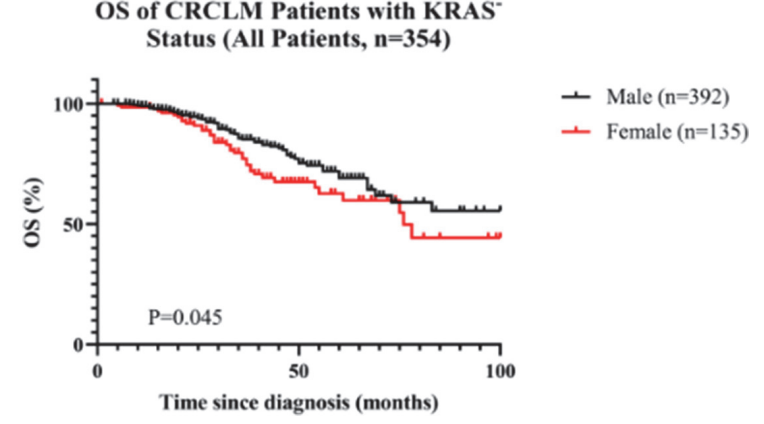

Time since diagnosis (months)

Abstract IDDF2021-ABS-0191 Figure 3 
A OS of CRCLM Patients Yonger than

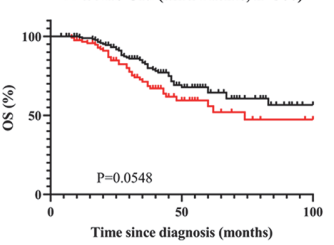

B OS of CRCLM Patients Aged from 45 to 74

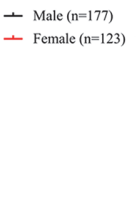

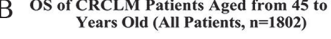

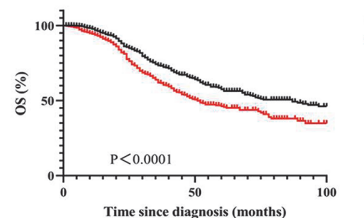

C OS of CRCLM Patients Older than — Male (n=1146)
— Female (655)

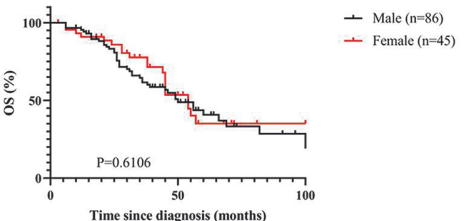

Abstract IDDF2021-ABS-0191 Figure 4

istics and outcome were extracted manually. Overall survival (OS) was analyzed using Kaplan-Meier method. Cox proportional hazard regression was performed to control the prognostic variables.

Results Of 4912 patients with CRCLM in our cohort, 2956 $(60.12 \%)$ were male. Comparing with male patients, females showed a significantly higher frequency of extrahepatic metastasis $(28.2 \%$ vs. $19.8 \%, \mathrm{P}<0.0001)$. Moreover, male had better 8-year OS than female in both left and right colon cancer (Left: male $44.3 \%$ vs female $34.4 \%$; $\mathrm{P}=0.0001$; Right: male $51.9 \%$ vs. female $39.5 \%, \quad \mathrm{P}=0.0004$ ) (IDDF2021-ABS-0191 Figure 1A, IDDF2021-ABS-0191 Figure 1B). Similarly, in both simultaneous and metachronous liver metastasis males also showed a better 8-year OS (simultaneous: male $44.0 \%$ vs. female 36.9\%, $\mathrm{P}<0.0001$; metachronous: male $53.3 \%$ vs. female 41.1\%, $\mathrm{P}=0.0006$ ) (IDDF2021-ABS-0191 Figure 2A, IDDF2021-ABS-0191 Figure 2B). Among patients with KRAS mutant status or age ranged from 44 to 74 years old, males also showed a favorable 8-year OS (IDDF2021-ABS-0191 Figure 3A, IDDF2021-ABS-0191 Figure 3B, IDDF2021-ABS-0191 Figure 4A, IDDF2021-ABS-0191 Figure 4B, IDDF2021-ABS0191 Figure4C).

Conclusions The advantage of males in survival indicates the impact of sex disparity in CRCLM. Further investigation in regard of the gender differences in CRCLM is warranted to investigate the potential mechanisms.

\section{IDDF2021-ABS-0192 THE ROLE CYTOMEGALOVIRUS DETECTION IN ACTIVE INFLAMMATORY BOWEL DISEASE}

${ }^{1}$ John Shao-Rong Mok*, ${ }^{1}$ Bernice Tan, ${ }^{2}$ Ee Jin Goh, ${ }^{1}$ Maimouna Nasser Khalifa Mandhari, ${ }^{1}$ Calvin Jianyi Koh, 'Jonathan Wei Jie Lee, 'Michelle Gowans, 'David Ong, 'Juanda Leo Hartono. 'Division of Gastroenterology and Hepatology, National University Hospital, Singapore; ${ }^{2}$ University of Auckland, New Zealand

\subsection{6/gutjnl-2021-IDDF.173}

Background It is known that Cytomegalovirus (CMV) can be detected in the colon during active Inflammatory Bowel Disease (IBD). However, its pathogenic role in causing active inflammation remains unclear as this ubiquitous virus is also regarded as innocent bystander. We aim to examine the utility of CMV testing in the colonic specimen and correlate with clinical outcome.

Methods A retrospective review of IBD patients with active symptomatic disease undergoing colonoscopy in National University Hospital Singapore from 2012-2020 and CMV tissue studies (histology with CMV Immunohistochemistry (IHC), CMV polymerase chain reaction (PCR), and tissue CMV culture) was conducted. The electronic medical record was analysed for clinical outcomes and CMV treatment.

Results Of 492 patients under IBD clinic follow up, 91 patients with active disease (42 Crohn's Disease, 44 Ulcerative Colitis, 5 Unclassified IBD) underwent colonoscopy and CMV tissue studies. The mean age is $40.13 \pm 15.24$ years with 54 males and 37 females. CMV tissue studies were positive in $20(28.8 \%)$ patients. A large majority of these patients $(14 / 20 ; 70 \%)$ achieved steroid-free remission without CMV treatment which suggests that CMV is innocent bystander. However, the rest (6 patients) had worsening or protracted active disease and were treated with a course of valganciclovir or ganciclovir which resulted in remission in all 6 patients, suggesting that CMV contributed to active disease activity.

We compared different testing modalities among those 20 patients with positive CMV and correlated with disease course. Refractory disease occurred in $2 / 3(66.6 \%)$ IHC positive patients and $3 / 5(60.0 \%)$ patients with positivity of both IHC and CMV PCR, as compared to only 1/11 (9.1\%) patients with positive CMV PCR, and none $(0 / 1)$ in patients with positive CMV culture.

Conclusions Colonic CMV was detected in $28.8 \%$ of patients with active IBD, but the large majority does not need CMV treatment. Positive IHC was associated with refractory disease as compared to PCR and CMV cultures.

\section{IDDF2021-ABS-0206 A NEW COMPUTER-ASSISTED DIAGNOSIS SCORING SYSTEM BASED ON DEEP LEARNING FOR PREDICTING INFLAMMATORY ACTIVITY FROM PATIENTS WITH ULCERATIVE COLITIS}

${ }^{1}$ Hongzhi $\mathrm{Xu}^{*},{ }^{1}$ Yanyun Fan, ${ }^{2}$ Ruochen Mu, ${ }^{2}$ Yinghao Zhang, ${ }^{3}$ Chenxi Xie, ${ }^{3}$ Lupeng Liu, ${ }^{3}$ Lin Wang, ${ }^{1}$ Jianlin Ren, ${ }^{2}$ Liansheng Wang. 'Department of Gastroenterology, Zhongshan Hospital Affiliated to Xiamen University, China; ${ }^{2}$ Department of Computer Science, Xiamen University, China; ${ }^{3}$ Department of Digestive Diseases, School of Medicine, Xiamen University, China

\subsection{6/gutjpl-2021-IDDF.174}

Background Endoscopy is increasingly important for the evaluation of patients with ulcerative colitis (UC). However, there were considerable differences in endoscopic assessment because of the endoscopists' training experience, not only that, existing endoscopy scoring methods cannot reflect the inflammation's details of the full-length endoscopic video; therefore, we sought to develop an automatic scoring system using deep learning technology for consistent and objective of endoscopic images and videos from patients with UC. 